\title{
Numerical Matching During Cerebellar Development: Quantitative Analysis of Granule Cell Death in Staggerer Mouse Chimeras
}

\author{
Karl Herrup and Karen Sunter \\ Department of Human Genetics, Yale Medical School, New Haven, Connecticut 06510
}

Cell death is a common yet puzzling feature of the development of many populations of neurons in the CNS. In the invertebrate phyla, such death is often preprogrammed; by contrast, in vertebrates, the best studied examples of histogenetic cell death are influenced by interactions among the neurons and their target. One attempt to explain this seemingly wasteful scheme of development has led to the hypothesis that this target-related cell death allows 2 populations of cells, which develop in isolation, to come into numerical and functional balance and hence to provide an epigenetic "buffer" mechanism to accommodate developmental variations. In the current study we have examined the extent to which the cell death observed in the cerebellar granule cell population serves to numerically match these neurons with their primary postsynaptic target, the Purkinje cell. Staggerer chimeras were made by aggregating 8-cell staggerer embryos with embryos of wild-type genotype. The cerebella of the resulting animals developed with widely varying numbers of normal (wild-type) Purkinje cell targets. Although staggerer Purkinje cells were present in the chimeric brains, these cells are intrinsically deficient in their normal developmental program (in the mutant, because of this deficiency, $100 \%$ of the granule cells die). Both granule cells and Purkinje cells were counted in chimeras and several wild-type mice. The results reveal that the number of granule cells present in these brains has a linear relationship with the number of Purkinje cells, and that the line connecting the points intersects the $Y$-axis close to the origin. These observations suggest that numerical matching is an important function of target-related cell death in the granule cell population. As a consequence of this process, neuron and target come into an accurate, predetermined numerical balance. The results are further discussed within the context of the control of cell number in the mammalian CNS.

One of the greatest paradoxes in the development of the nervous system is the phenomenon of naturally occurring cell death. During embryogenesis, when the goal of the developing organ-

\footnotetext{
Received May 30, 1986; revised Aug. 22, 1986; accepted Aug. 26, 1986.

We wish to acknowledge the financial support of the March of Dimes Birth Defects Foundation through the award of a Basic Research Grant (7-163) and the NIH (NS-20591). We are also grateful to the laboratory of David Van Essen of the Division of Biology at the California Institute of Technology for allowing the use of his computerized microscope system to create the reconstructions shown in Figure 5. Finally, K.H. would like to acknowledge the Comelius V. Wiersma fund for sponsoring his leave at Caltech and to extend his gratitude to James Bower for opening his lab to an East Coast visitor. It was during this period that most of the writing of the manuscript was accomplished.

Correspondence should be addressed to Karl Herrup, Department of Human Genetics, Yale Medical School, 333 Cedar Street, New Haven, CT 06510.

Copyright (C) 1987 Society for Neuroscience $0270-6474 / 87 / 030829-08 \$ 02.00 / 0$
}

ism would seem to be growth and addition of cells, many neural centers in both the CNS and PNS pass through a period in which a substantial fraction of the population becomes necrotic and dies (for recent reviews, see Cunningham, 1982; Hamburger and Oppenheim, 1982; Cowan et al., 1984; Lamb, 1984; Oppenheim, 1985). Yet, while the fact of its existence is well established, both the mechanism by which the cell death process is controlled and the developmental "logic" behind its occurrence remain a mystery. One rationale frequently employed to explain this paradox is that cell death represents a mechanism for control of cell number during development. The idca is that the process of cell death allows 2 populations that are anatomically connected in the adult but spatially separate in the embryo (e.g., nerve and muscle) to adjust during development in such a way that their numbers are appropriately balanced.

In part, this concept has developed because of the experimental observation that the amount of cell death in a population is related to the presence or absence of the target cells of that population. In most systems studied the removal of the target leads to a dramatic increase in the amount of cell death and a concomitant decrease in the size of the adult population. If target is completely removed early enough in development, then virtually all of the presynaptic neurons will degenerate (Lamb, 1981; Lanser and Fallon, 1984). Though more difficult to achieve, increasing the target-to-neuron ratio has been shown to decrease the amount of observed cell death. Hollyday and Hamburger (1977) achieved a 10-30\% increase in the number of surviving chick motor neurons by transplanting an extra limb. Narayanan and Narayanan (1978) observed a similar reduction of cell death in the chick ciliary ganglia, accessory oculomotor nuclei, and trochlear nucleus after transplanting an extra optic primordium. Pilar et al. (1980) achieved an increase in target-to-neuron ratio by decreasing the normal neuronal complement by half and leaving the target intact. They too observed a reduction in the amount of cell death in the remaining neurons.

From these semiquantitative observations has grown the concept that target-related cell death performs a numerical matching function (Hamburger, 1975; Katz and Lasek, 1978). The implication of this hypothesis is that there must be some numerical relationship that allows the definition of presynaptic neuron number as a function of target size. A beginning attempt to describe this function for the lateral motor column has been made by McLennan (1982) and by Tanaka and Landmesser (1986). Tanaka and Landmesser (1986), in particular, have shown, by transplanting chick limbs to quail embryos and vice versa, that a high degree of correlation is found between postcell-death neuron number and target size (measured as number of target myotubes). While only 4 points were available for graphing, these 4 fit a straight-line function well. 
Our laboratory has been interested in the regulation of cell number in the CNS for some time. We have used as our model mouse cerebellar cortex and those factors that regulate both Purkinje and granule cell number. The granule: Purkinje cell contact is analogous to the model systems described above for several reasons. First, Caddy and Biscoe (1979) have shown that a reduction of granule cell number occurs during normal cerebellar development. The reduction amounts to approximately $20-30 \%$ of the initial population. Second, the effects of target removal can be documented by a series of neurological mutations that block the ability of granule cell axons to make appropriate contact with Purkinje cells (see Sidman, 1968, 1972, 1983; Sotelo, 1975; Mullen and Herrup, 1979, for review). Third, comparisons among mutants that block this contact before (staggerer: Landis and Sidman, 1978; Herrup and Mullen, 1979a, 1981), during (lurcher: Caddy and Biscoe, 1979; Wetts and Herrup, 1982a, b), and after (Purkinje cell degeneration: Mullen et al., 1976) the bulk of synaptogenesis has occurred suggest very strongly that, as with the neurons of the lateral motor column and the ciliary ganglion, there is a critical period for this interaction as well.

In the present study, we have used the cerebellum and the granule : Purkinje cell circuit as a model system to explore the process of target-related cell death. Rather than alter the size of the target by surgery or drugs, we have used a genetic mutation known as staggerer (gene symbol, $s g$ ). In this mutant, the Purkinje cells never develop the tertiary dendritic spines that are the site of granule cell : Purkinje cell synapse, and hence the granule cells never have a competent target with which to interact (Sidman, 1968; Sotelo and Changeux, 1974; Yoon, 1979ac). Experimental aggregation chimeras made between $\mathrm{sg} / \mathrm{sg}$ and $+/+$ embryos were used to vary the number of potential target cells over a wide range. The results of our quantitative analysis illustrate that, in the absence of other variables, the function that defines the plot of granule cell number versus Purkinje cell number is a straight line. This finding strongly supports the concept that one of the functions of target-related cell death is the numerical matching of pre- and postsynaptic neuronal populations.

\section{Materials and Methods}

Animals. Mice used as cmbryo donors in this study were primarily received directly from the Jackson Laboratory (Bar Harbor, ME). The staggerer mutants, however, were all bred in our colony at the Yale Medical School. They are currently being maintained on a background that is $\mathrm{N} 7$ on $\mathrm{C} 57 \mathrm{BL} / 6 \mathrm{~J}$ and has been further inbred for 5 generations. The original stock was received from the Jackson Laboratory and includes 2 genetic marker genes in repulsion to staggerer on chromosome 9: dilute (d) and short ear (se). Males and females of genotype ( $s g++/+d s e$ ) are intercrossed, and the genotypes of the resulting pups are distinguishable by $15 \mathrm{~d}$ of age either by coat color (the dilute gene results in a gray coat) or by behavior. Animals are maintained on a 12-hr light/dark cycle with food and water ad libitum.

Chimeras were made by standard protocols (Tarkowski, 1961; Mintz, 1962, 1965; Mullen and Whitten, 1971). The resulting animals were identified as to their genotype mostly on the basis of cerebellar morphology. If no Purkinje cells from the ?/sg embryo were wild type in appearance, the chimera was assumed to be $s g / s g \leftrightarrow+/+$ in genotype. This conclusion was most often confirmed by an overall reduction in cerebellar size (see Fig. 4).

Histology and cell counts. Preparation of histological and histochemical material was as described previously (Mullen, 1977; Herrup and Mullen, 1979b; Herrup and Sunter, 1986). The chimeras described here were all constructed with 1 staggerer embryo (on a $\beta$-glucuronidase background that is $G u s^{b} / G u s^{b}$ ) and 1 wild-type embryo from a strain whose $\beta$-glucuronidase background was $G u s^{h} / G u s^{h}$. The only exception to this was $\chi 96$, whose wild-type component was from the IC/Le strain and who was partially analyzed previously as part of a different study (Herrup, 1983).

Purkinje cell counts were performed as described in earlier studies (e.g., Wetts and Herrup, 1982c; Herrup and Sunter, 1986). Granule cells were counted by protocols described by Wetts and Herrup (1983). Cerebellar granule cell layer areas were measured from sections approximately $160 \mu \mathrm{m}$ apart using a semiautomatic image analysis system (Bioquant, R\&M Biometrics, Nashville, TN). The resulting areas were graphed as function of distance from the midline, and the area under this curve was used to determine the granule cell layer volume. At $1000 \times$, random granule cell fields were examined, superimposed on a grid (seen through a drawing tube) that was $50 \mu \mathrm{m}$ on a side. A minimum of 36 such grid counts were done from each cerebellar half, representing all mediolateral and anterior-posterior regions of the cerebellum. As the section thickness was $8 \mu \mathrm{m}$, the average number of granule cells per grid was taken to be the number of cells in a volume of $20,000 \mu \mathrm{m}^{3}$. The total granule cell layer volume was divided by the grid volume and multiplied by the number of granule cells per grid. This value, which we term the raw count, was corrected by the method of Hendry (1976) to correct for split cells. Granule cell diameters were also measured on the Bioquant at $1000 \times$

\section{Results}

\section{Background on the staggerer mutation}

As reported previously, the staggerer mutation $(\mathrm{sg} / \mathrm{sg})$ results in the severe atrophy of the cerebellar cortex (Fig. 1). The midline cross-sectional area is dramatically reduced (Fig. 1, $A, B$ ), and the cells that remain in the mutant cortex (Fig. 1D) are markedly different from the wild type (Fig. 1C). Virtually $100 \%$ of the granule cells degenerate, primarily during the second to fourth postnatal weeks (Sidman et al., 1962). Although Purkinje cells are present, they are far from normal (Sidman, 1968; Bradley and Berry, 1978; Landis and Sidman, 1978). The best description of the mutant defect to date is that "the Purkinje cells are generated at the normal time, but are much delayed in postnatal differentiation, showing a remarkably immature form" (Sidman, 1968). The feature of this mutation that is most relevant for the current study is that the $s g / s g$ Purkinje cells lack the tertiary branchlet spines that serve as the site of synapse between the granule and Purkinje cells. While the dendrite of the normal Purkinje cell is studded with spines, the staggerer Purkinje cells have a withered dendritic arbor (see Golgi impregnations of Sidman, 1968; Bradley and Berry, 1978). This defect has been confirmed at the ultrastructural level (Sotelo and Changeux, 1974; Yoon, 1977a, b; Landis and Sidman, 1978).

In the mosaic cerebellum of the staggerer $\leftrightarrow$ wild-type chimera, Herrup and Mullen (1979b, 1981) showed that all of the Purkinje cell defects visible in the light microscope are intrinsic to the $s g / s g$ Purkinje cells themselves. That is, even in the prescnce of genctically wild-typc cclls, all staggerer cells retain the mutant phenotype of reduced number, small size, predominantly ectopic location, and regional variability. Because of the unavailability of appropriate cell markers, it is presumed, though never directly proven, that the tertiary branchlet spines are also absent. By contrast, the granule cells are not direct sites of gene action. Herrup (1983) showed that millions of genetically staggerer granule cells could be rescued in the mosaic environment of the chimeric cerebellum, presumably by the presence of wildtype Purkinje cells. Since the staggerer Purkinje cells never present a target to the developing granule cells and since the genetically mutant granule cells are capable of rescue and appear morphologically normal in the adult (Fig. 2, $B-D$ ), the staggerer chimeras are an ideal system in which to test the numerical matching hypothesis. The adequacy of this model is further confirmed by the work of Sonmez and Herrup (1983) who showed 

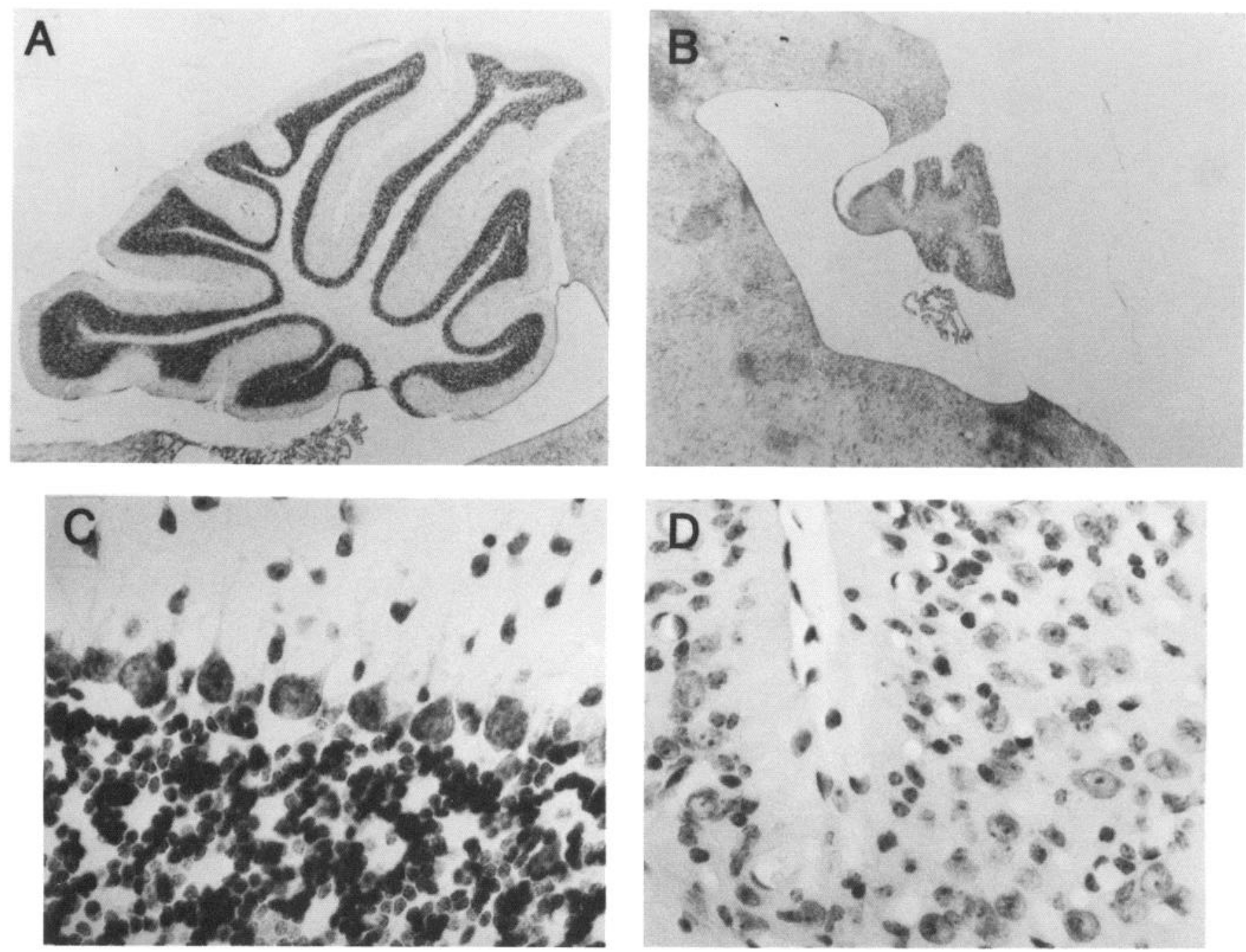

Figure 1. Comparison of cerebellar morphologies in wild-type $(A, C)$ and staggerer $(B, D)$ mice. $A$ and $B$, Sagittal sections near the midline from wild-type $(A)$ and staggerer $(B)$ cerebella. Note the dramatic reduction of cerebellar mass in the mutant. $C$ and $D$, Higher-magnification views of the cellular composition of wild-type $(C)$ and staggerer $(D)$ cerebellar cortex. By this age ( 3 months), the mutant has lost virtually all of its granule cells, although cells of basket, stellate, and glial morphology remain. The medium to large neurons (MLN) that remain are smaller than wild type and are not aligned in a single row. Original magnification: $A$ and $B, \times 12.5 ; C$ and $D, \times 500$.

that, in the mosaic brains of 16- to 17-d-old staggerer chimeras, the amount of cell death is intermediate between staggerer and wild type. Thus, cell death is an important factor in the regulation of final granule cell number in the chimeric cerebellar cortex.

\section{Identification of wild-type cells}

Purkinje cells were counted as described in Materials and Methods. The expected precision of these counts is not as high as that of other studies from this laboratory. To count a cell as present in a section, it must have a portion of its nucleus visible. In our material, this is best revealed by a Nissl stain such as cresyl violet (Fig. $2 \mathrm{C}$ ). In the counts of the staggerer chimeras, however, we also wish to count only wild-type Purkinje cells, as these are the cells that are presumably providing target for the granule cells. We therefore also need to know the genotype of each cell since staggerer Purkinje cells survive in the chimera (Mullen and Herrup, 1979; Herrup and Mullen, 1979b, 1981). This is revealed with certainty only through $\beta$-glucuronidase histochemistry, with which the mutant cells appear stained (red), while the wild-type cells appear unstained except with the methyl green counterstain (Fig. 2, $B-D$ ). Difficulties arise because, although most $\mathrm{sg} / \mathrm{sg}$ Purkinje cells in the chimera are ectopic (e.g., Fig. 2, B, C), some will occasionally be found in or near the true Purkinje cell layer (Fig. 2D). These cells might easily be mistaken for $+/+$ in cresyl violet preparations. To circumvent this difficulty we have adopted the convention of Sonmez and Herrup (1983). Any cell at the interface of the granule cell and molecular layers was counted as a wild-type Purkinje cell if it was sufficiently large in size.

\section{The staggerer chimeras}

Midline sagittal sections of the chimeras used in this study are shown in Figure 3. A wild-type animal is shown in Figure $3 \mathrm{~A}$ and a staggerer mutant in Figure $3 K$ for comparison. As illustrated by this montage, the ratio of the staggerer to wild-type composition varies widely. Cerebellar Purkinje cell counts from these animals are shown in Table 1. As might be predicted from the midsagittal areas, the number of surviving wild-type Purkinje cells varies from near wild-type values (e.g., chimera 264) 

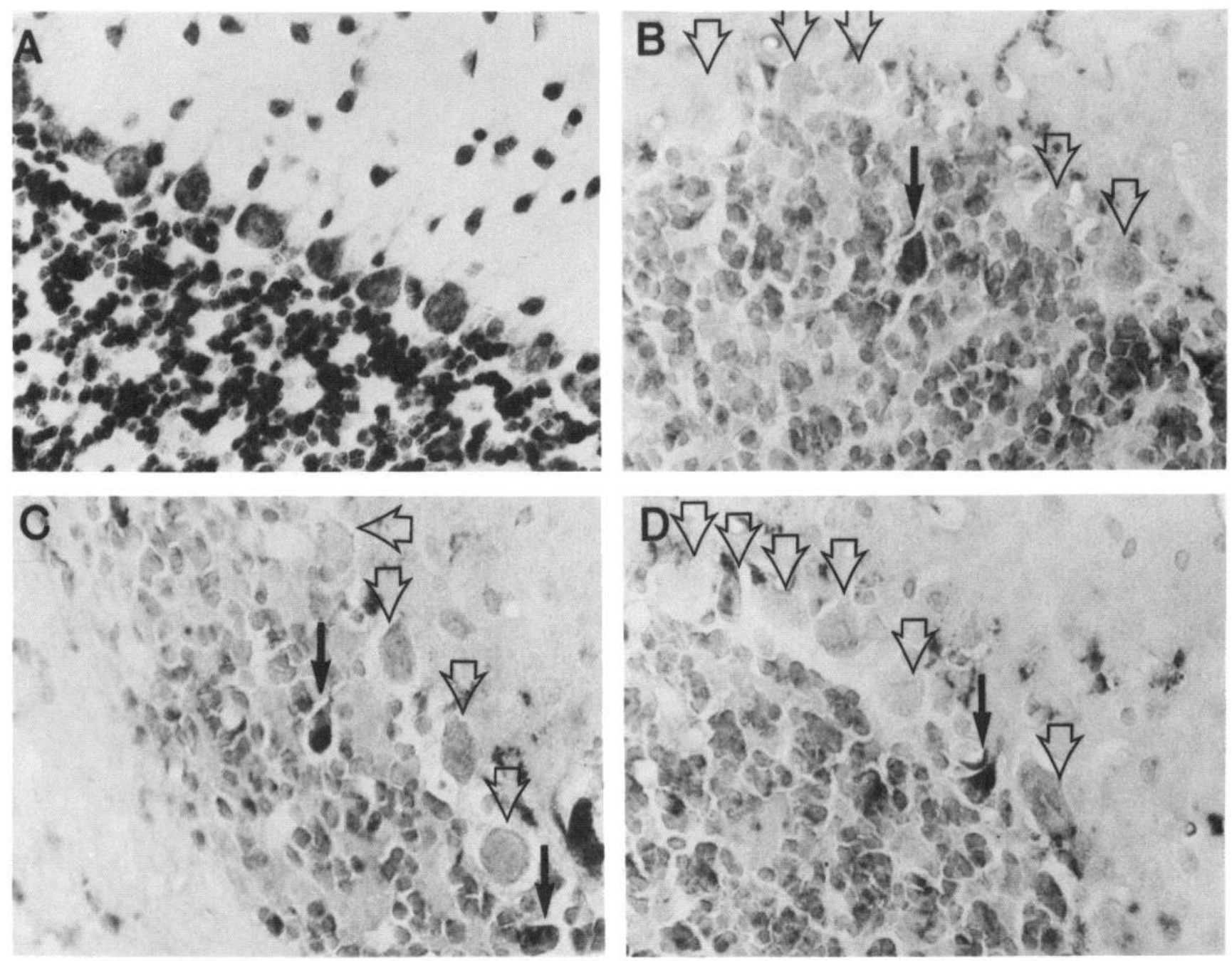

Figure 2. Appearance of the cerebellar cortex of wild-type $(A)$ and staggerer chimeric animals $(B-D)$. The wild-type section has been stained with cresyl violet; the chimera sections are all stained for $\beta$-glucuronidase histochemistry and counterstained with methyl green. In $B-D$, all $+/+$ Purkinje cells have been marked with open arrows, while the genetically $\mathrm{sg} / \mathrm{sg}$ cells have been marked with solid arrows. Although in most regions the $s g /$ $s g$ cells are ectopic in location (as well as small in size), they are occasionally found in or near the Purkinje cell layer as in $D$. If this $s g / s g$ cell were stained only with cresyl violet it could easily be mistaken for a $+/+$ cell.

to near mutant values (e.g., chimera 96). This variation was also apparent in the coats and livers of these animals (data not shown).

The distribution of the wild-type Purkinje cells in a representative sagittal section from $\chi 96$ is shown in Figure $4 A$. The position of each wild-type cell is noted by the presence of a dot. Note that the wild-type cells do not occur in large patches. Rather, they appear uniformly distributed across the entire reduced length of the Purkinje cell layer, especially in single sagittal sections. In addition, their density is reduced compared to wild type (Fig. 4B). An apparently uniform distribution has been reported previously using either GPI (Oster-Granite and Gearhart, 1981), $\beta$-glucuronidase (Mullen, 1978), pcd (Mullen, 1977), or lurcher (Wetts and Herrup, 1982c) as a cell marker. This observation is important for the interpretation of this study as it means that the variation in target size is not occurring to vastly different extents in different cerebellar regions.

Granule cells were counted as described in Materials and Methods. Since it is not necessary to know the genotype of a granule cell (neither genotype is intrinsically altered; Herrup, 1983), cresyl violet material was used exclusively. Table 1 shows the numbers of granule cells surviving in each of the half-cerebella examined in this study. In addition, some of the salient quantitative data from our determinations of granule cell layer volume and granule cell density are reported. To test the numerical matching hypothesis using the granule: Purkinje cell circuit as a model, it is assumed that Purkinje cell number is the independent variable, while granule cell number is the dependent variable. Figure 5 shows the resulting graph. Each filled circle represents the counts of 1 hemi-cerebellum from 1 staggerer $\leftrightarrow$ wild-type chimera. The points suggest a linear relationship, and the solid line is the least-squares fit of the chimera data plus the staggerer mutant (zero Purkinje cells, zero granule cells). The equation of this line $(y=m x+b)$ is granule cell number $=192 \times$ Purkinje cell number $+1.17 \times 10^{6}$. The correlation coefficient $\left(r^{2}\right)$ is 0.969 .

\section{Discussion}

The numerical relationship between cerebellar granule cells and Purkinje cells in staggerer chimeric mice has been examined as a model system in which to study the phenomenon of naturally 

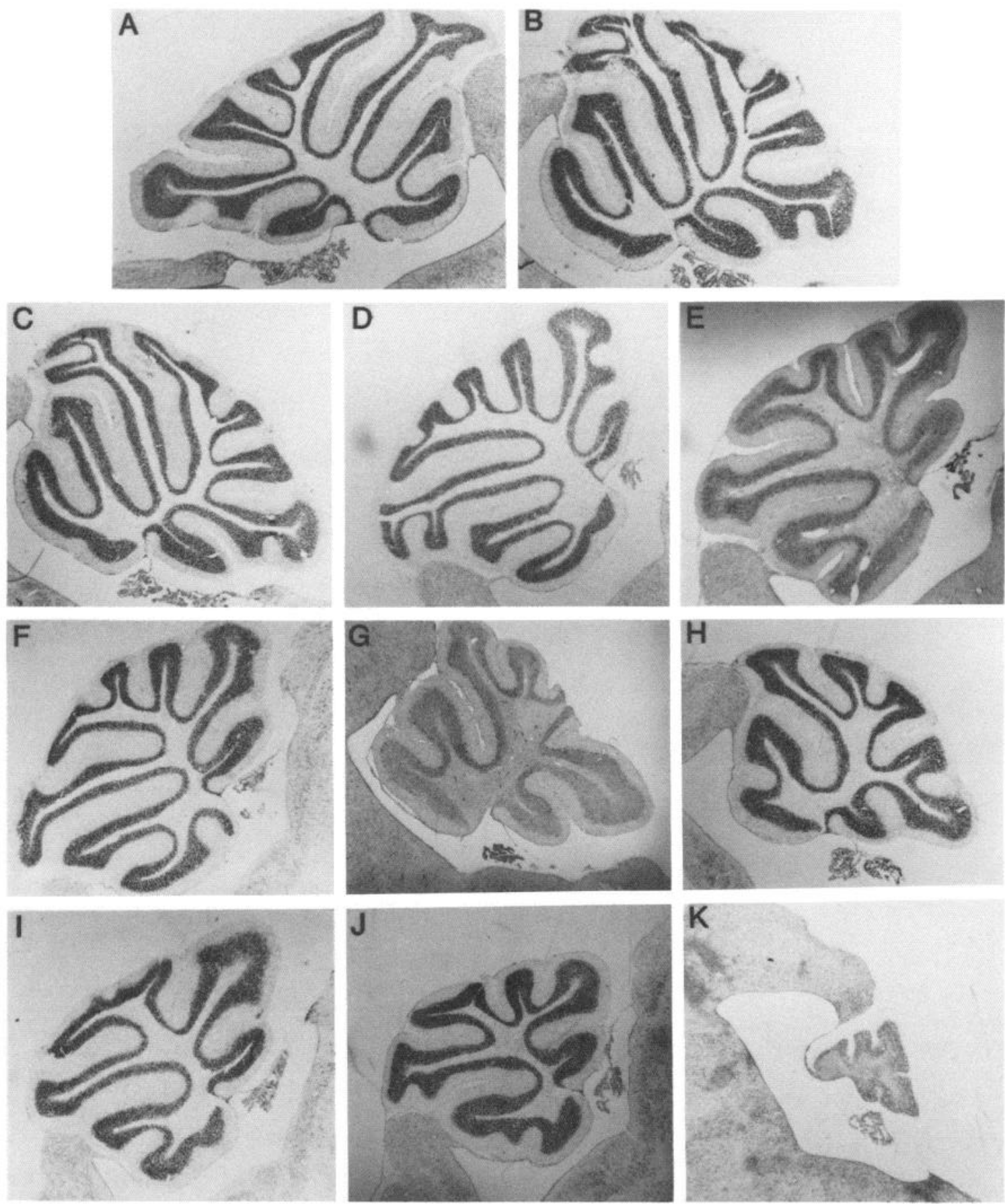

Figure 3. Midsagittal sections from the chimeras analyzed in this study. These micrographs emphasize the ability to achieve a finely graded series of cerebellar morphologies ranging from small (with many fewer granule and Purkinje cells) to near wild-type in size. The cerebellum in $A$ is from a wild-type C57BL/6J mouse; the cerebellum in $K$ is from a homozygous staggerer mutant. Both animals were 3 months of age. The remaining micrographs in $B-J$ are from $\chi 264, \chi 263, \chi 280, \chi 2, \chi 159, \chi 1, \chi 238, \chi 168$, and $\chi 96$, respectively.

occurring cell death during the development of the mammalian CNS. Chimeras represent an attractive model system for the study of cell death for several reasons. First, the site of gene action can be defined to such a level as to assure that the only direct effect of the gene is on the target cell (i.e., the Purkinje cell). Second, in the chimeras, the Purkinje cells that remain have been untreated by drugs or radiation and unperturbed by surgical intervention during development. The genetics serve as a precision neurological tool to remove only Purkinje cells. Third, the target population is not removed in big chunks. Rather, there is a diffuse loss of cells throughout the cerebellum as illustrated in Figure 4 (see also Wetts and Herrup, 1982a, c; as well as Mullen, 1977, for $p c d / p c d \leftrightarrow+/+$ chimeras and OsterGranite and Gearhart, 1981, for Gpi-1a/Gpi-1 $1^{a} \leftrightarrow G p i-1^{b} / G p i-1^{b}$ chimeras). Fourth, the fact that the genotype ratio (mutant to wild type) can vary widely from 0 to $100 \%$ means that a broad distribution of points can be examined. Finally, the nature of the system is such that the Purkinje cells that remain and serve 

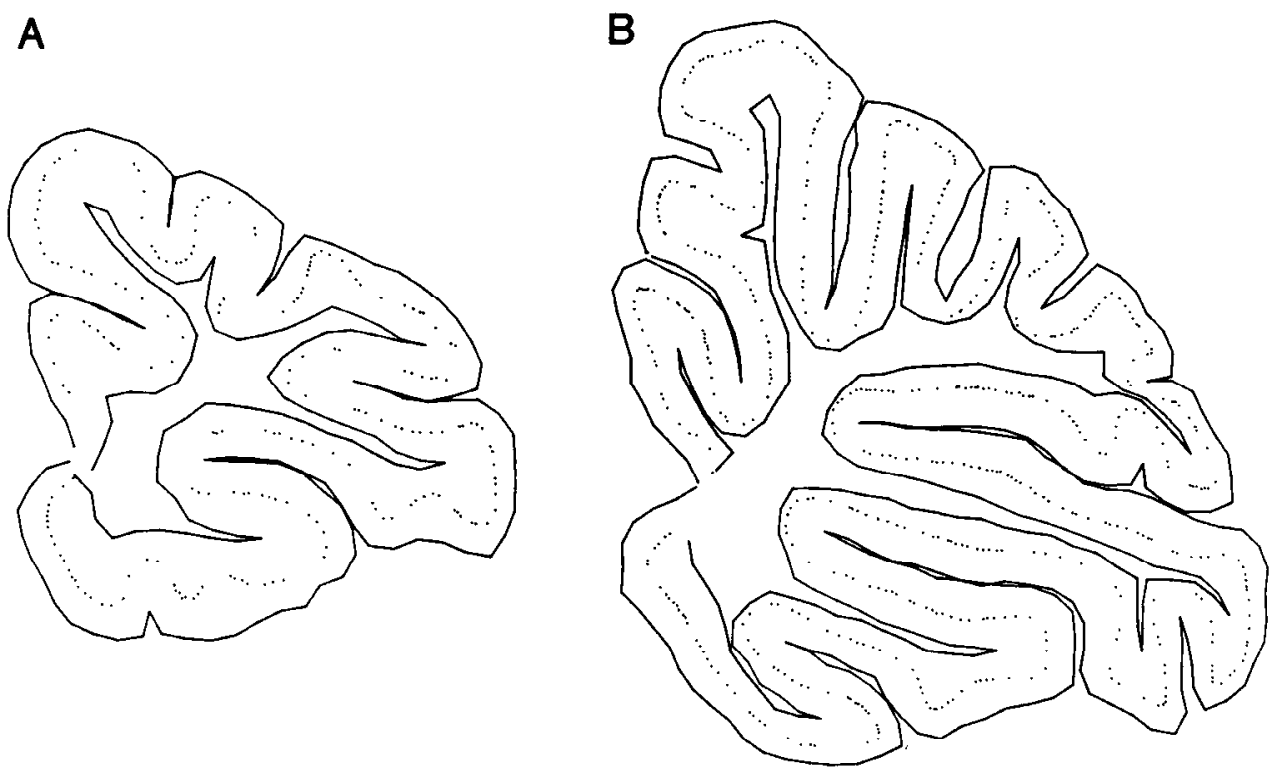

Figure 4. Reconstruction of the Purkinje cell layer of $\chi 96(A)$ and a wildtype $(B)$ mouse. Both sections are from near the midline. In each the cerebellar surface and inner edge of the internal granule cell layer have been drawn in. The position of each normal Purkinje cell has been noted by the presence of a single dot. Note that while there is an overall reduction in the length and density of the Purkinje cell layer in the chimera, there are no large regions where the Purkinje cells are totally absent. Thus, granule cell target is being removed diffusely in the chimeras.

as the focus of the study are all genetically wild type. Thus, undetected pleiotropic effects of the mutation are not likely to interfere with the analysis.

Although the genetic approach is quite distinct from previous studies of experimentally manipulated cell death (in which increases and decreases of target size were achieved primarily through surgical manipulation of target or presynaptic population size), our results are in complete agreement with the results and predictions of these earlier studies. Both McLennan (1982) and Tanaka and Landmesser (1986) found a good correlation between the number of surviving motor neurons in the spinal cord and the number of target myotubes present during the cell death period. The latter authors suggested, as we do, that a straight-line relationship exists between the size of the presynaptic neuron population and its target.

Our data confirm and extend these observations. The ratio of granule cells to Purkinje cells in our various experimental animals remains nearly constant despite a wide variation in the number of wild-type Purkinje cells present as granule cell targets. Thus, when the data are plotted as in Figure 5, a straight-line relationship is the best fit to the data. The significance of this observation is made greater by the fact that the points determined from the staggerer chimera data almost perfectly extend the line defined previously (Wetts and Herrup, 1983) by various inbred (nonchimeric) strains of laboratory mice (the open symbols in Fig. 5). Thus, the relationship we are describing here is not an artifact of either the staggerer gene or the chimeric nature of the tissue we are analyzing.

It is informative to compare the linear relationship observed in the present study of staggerer chimeras with the curvilinear relationship described by Wetts and Herrup (1983). The 2 studies are virtually identical in their approach and choice of model system. What distinguishes them is the mutation used to eliminate the Purkinje cell target. Wetts and Herrup (1983) used the lurcher mutation to destroy all genetically $+/ L c$ Purkinje cells in the chimeric cerebella. However, since $+/ L c$ Purkinje cells do not die until the granule cells have already begun forming synapses with them, these authors hypothesized that the curvilinear relationship might be due to this early presence of $+/ L c$ Purkinje cells in the chimeras. These transient neurons, while potentially contributing to granule cell survival, would be gone before the animals were sacrificed, and hence their numerical presence would go undetected in the counts of adult animals. By contrast, the staggerer mutation results in Purkinje cells that are reduced in number and have atrophic dendritic arbors that never develop tertiary branchlet spines, the site of granule cell parallel fiber synapse. These mutant Purkinje cells thus intrinsically lack any granule cell target. These properties mean that the wild-type cells that we count in the adult chimeras are an accurate representation of the quantity of postsynaptic target available to the developing granule cells during growth and synaptogenesis.

The implication of the linear relationship is that if the variable of target size is isolated as the independent variable, then numerical matching does indeed occur between Purkinje and granule cells, i.e., the granule cell population uses a strict developmental algorithm to adjust its numbers to the size of the

Table 1. Quantitative features of the granule cell layers of the $s g / s g \leftrightarrow+/+$ chimeras used in this study

\begin{tabular}{llclc} 
Chimera & $\begin{array}{l}\text { Granule } \\
\text { cell layer } \\
\text { volume } \\
\left(\times 10^{9} \mu \mathrm{m}^{3}\right)\end{array}$ & $\begin{array}{l}\text { Granule } \\
\text { cell density } \\
(\text { per } 20,000\end{array}$ & $\begin{array}{l}\text { Granule } \\
\text { cell number } \\
\left(\times 10^{6}\right)\end{array}$ & $\begin{array}{l}\text { Purkinje } \\
\text { cell } \\
\text { number } \\
\left(\times 10^{3}\right)\end{array}$ \\
\hline$\chi 263$ left & 4.40 & 89.2 & 18.1 & 91.1 \\
$\chi 263$ right & 4.70 & 107.1 & 19.2 & 100.1 \\
$\chi 238$ left & 2.87 & 101.8 & 10.4 & 47.1 \\
$\chi 238$ right & 2.77 & 90.5 & 9.10 & 46.8 \\
$\chi 264$ left & 5.08 & 114.2 & 22.4 & 103.4 \\
$\chi 264$ right & 4.70 & 114.5 & 19.7 & 96.5 \\
$\chi 280$ left & 4.42 & 120.4 & 20.9 & 86.1 \\
$\chi 280$ right & 4.17 & 112.0 & 19.0 & 92.3 \\
$\chi 168$ left & 2.53 & 107.0 & 9.43 & 53.2 \\
$\chi 168$ right & 2.46 & 103.2 & 7.85 & 39.1 \\
$\chi 159$ left & 3.56 & 111.6 & 14.0 & 81.0 \\
$\chi 96$ left & 2.75 & 97.0 & 10.4 & 26.7 \\
$\chi 1$ left & 3.50 & 94.5 & 12.1 & 57.5 \\
$\chi 2$ left & 4.20 & 94.3 & 8.09 & 34.5 \\
\hline
\end{tabular}




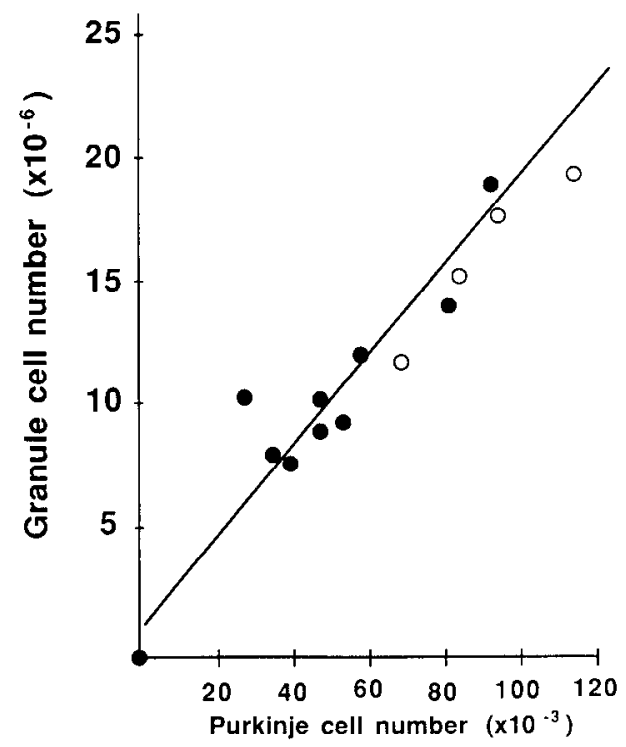

Figure 5. Counts of cerebellar granule cells as a function of $+/+$ Purkinje cell number in wild-type and staggerer mice and their chimeras. The wild-type data (from Wetts and Herrup, 1983) have been plotted as open circles, the staggerer chimeras data as filled circles. The single point at the origin represents the staggerer mutant, with no granule cells and no $+/+$ Purkinje cells present. The straight line is the least-squaresfit of the chimera data plus the staggerer mutant. The slope of the line is 192 granule cells/Purkinje cell; the $Y$-intercept is $1.17 \times 10^{6}$ granule cells.

postsynaptic, Purkinje cell target population. The straight-line relationship suggests that there is very little, if any, "plasticity" in the interaction. A given Purkinje cell population seems capable of supporting only a specific number of granule cells, and it does so even when the number of granule cells needing such support is increased, as is the case in the chimeras. At the same time, a given granule cell population seems to require support from a relatively specific number of Purkinje cells and can do with no fewer, even in situations where competition is stiff. It is worth noting that while the preceding comments focus on cerebellar cortex, the same discussion applies equally to the results described in the lateral motor column. That these 2 different systems led 2 groups of investigators to the same conclusions suggests that the principle of numerical matching probably applies to many cases of target-related cell death throughout the nervous system.

It is informative to view these results in the broader context of intrinsic versus cxtrinsic control of cell number in the mammalian CNS. Previous studies from our laboratory (Wetts and Herrup, 1982a-c; Herrup et al., 1984; Herrup, 1986a, b; Herrup and Sunter, 1986) have demonstrated that the cerebellar Purkinje cells descend from a small number of progenitors selected in the early stages of CNS development to be the sole source of the adult population of these neurons. In addition, during normal, unperturbed development, each of these progenitor cells appears intrinsically programmed to produce a strain-specific number of adult Purkinje cells (i.e., the number of cells in the clone derived from one of the Purkinje progenitors is constant and characteristic of the genotype of the progenitor). From the standpoint of cell number control, once the number of progenitors has been selected, the size of the adult Purkinje cell population has been determined and is intrinsic to the lineages. Later, interactions between the granule and Purkinje cell pop- ulations rigorously adjust granule cell number to match this number of Purkinje cells. Thus, numerical decisions made in the earliest stages of CNS development, and executed through cell lineage relationships, can potentially specify cell numbers for many other interacting neuronal populations in the adult brain via the numerical matching process. These second-order specifications are extrinsic to the population, however, since they rely on cell:cell interactions, and thus are modifiable through changes in the developmental process in a given organism. The granule cell counts in our chimeras (with widely varying target sizes) illustrate that any system thus constructed has wide latitude for numerical response to unexpected developmental changes. These response potentials, however, are based on apparently strict but relatively simple rules.

\section{References}

Bradley, P., and M. Berry (1978) Purkinje cell dendritic tree in mutant mouse cerebellum. A quantitative study of weaver and staggerer mice. Brain Res. 142: 135-141.

Caddy, K. W. T., and T. J. Biscoe (1979) Structural and quantitative studies on the normal $\mathrm{C} 3 \mathrm{H}$ and Lurcher mutant mouse. Phil. Trans. R. Soc. Lond. 287: 167-201.

Cowan, W. M., J. W. Fawcett, D. D. M. O'Leary, and B. B. Stanfield (1984) Regressive events in neurogenesis. Science 225: 1258-1265.

Cunningham, T. J. (1982) Naturally occurring neuron death and its regulation by developing neural pathways. Int. Rev. Cytol. 74: 163186.

Hamburger, V. (1975) Cell death in the development of the lateral motor column of the chick embryo. J. Comp. Neurol. 160: 535-546.

Hamburger, V., and R. W. Oppenheim (1982) Naturally occurring cell death in vertebrates. Neurosci. Comment. 1: 39-48.

Hendry, I. A. (1976) A method to correct adequately for the change in neuronal size when estimating neuronal numbers following nerve growth factor treatment. J. Neurocytol. 5: 337-349.

Herrup, K. (1983) Role of staggerer gene in determining cell number in cerebellar cortex. I. Granule cell death is an indirect consequence of staggerer gene action. Dev. Brain Res. 11: 267-274.

Herrup, K. (1986a) Roles of cell lineage in the developing mammalian brain. In Neural Development (Curr. Top. Dev. Biol., Vol. 18), R. K. Hunt, ed., Academic, New York (in press).

Herrup, K. (1986b) Cell lineage relationships in the development of the mammalian CNS: Role of cell lineage in control of cerebellar Purkinje cell number. Dev. Biol. 115: 148-154.

Herrup, K., and R. J. Mullen (1979a) Regional variation and absence of large neurons in the cerebellum of the staggerer mouse. Brain Res. 172: $1-12$.

Herrup, K., and R. J. Mullen (1979b) Staggerer chimeras: Intrinsic nature of Purkinje cell defects and implications for normal cerebellar development. Brain Res. 178: 443-457.

Herrup, K., and R. J. Mullen (1981) Role of staggerer gene in determining cell number in the cerbellar cortex: A quantitative analysis of staggerer chimeric mice. Dev. Brain Res. 1: 475-485.

Herrup, K., and K. Sunter (1986) Lineage dependent and independent control of Purkinje cell number in the mammalian CNS: Further quantitative studies of lurcher chimeric mice. Dev. Biol. 117: 417427.

Herrup, K., A. Letsou, and T. J. Diglio (1984) Cell lineage relationships in the development of the mammalian CNS: The facial nerve nucleus. Dev. Biol. 103: 329-336

Hollyday, M., and V. Hamburger (1977) An autoradiographic study of the formation of the lateral motor column in the chick embryo. Brain Res. 132: 197-208.

Katz, M. J., and R. J. Lasek (1978) Evolution of the nervous system: Role of ontogenetic buffer mechanisms in the evolution of matching populations. Proc. Natl. Acad. Sci. USA 75: 1349-1352.

Lamb, A. H. (1981) Target dependency of developing motoneurons in Xenopus laevis. J. Comp. Neurol. 203: 157-171.

Lamb, A. H. (1984) Motoneuron death in the embryo. Crit. Kev. Clin. Neurobiol. 1: 141-179.

Landis, D. M. D., and R. L. Sidman (1978) Electron microscopic analysis of postnatal histogenesis in the cerebellar cortex of staggerer mutant mice. J. Comp. Neurol. 179: 831-863. 
Lanser, M. E., and J. F. Fallon (1984) Development of the lateral motor column in the limbless mutant chick embryo. J. Neurosci. 4 . 2043-2050.

McLennan, I. S. (1982) Size of motoneuron pool may be related to number of myotubes in developing muscle. Dev. Biol. 92: 263-265.

Mintz, B. (1962) Formation of genotypically mosaic mouse embryos. Am. Zool. 2: 432.

Mintz, B. (1965) Genetic mosaicism in adult mice of quadriparental lineage. Science 148: 1232-1233.

Mullen, R. J. (1977) Site of $p c d$ gene action and Purkinje cell mosaicism in cerebella of chimeric mice. Nature 272: 245-247.

Mullen, R. J. (1978) Mosaicism in the central nervous system of mouse chimeras. In The Clonal Basis of Development, 36th Symp. Soc. Dev. Biol., S. Subtelney and I. Sussex, eds., pp. 83-101, Academic, New York.

Mullen, R. J., and K. Herrup (1979) Chimeric analysis of mouse cerebellar mutants. In Neurogenetics: Genetic Approaches to the Nervous System, X. O. Breakefield, ed., pp. 271-297, Elsevier NorthHolland, New York.

Mullen, R. J., and W. K. Whitten (1971) Relationship of genotypes and degree of chimerism in coat color to sex ratios and gametogenesis in chimeric mice. J. Exp. Zool. 178: 165-176.

Mullen, R. J., E. M. Eicher, and R. L. Sidman (1976) Purkinje cell degeneration: A new neurological mutant in the mouse. Proc. Natl Acad. Sci. USA 73: 208-212.

Narayanan, C. H., and Y. Narayanan (1978) Neuronal adjustments in developing nuclear centers of the chick embryo following transplantation of an additional optic primordium. J. Embryol. Exp. Morphol. 44: 53-70.

Oppenheim, R. W. (1985) Naturally occurring cell death during neural development. Trends Neurosci. 8: 487-493.

Oster-Granite, M. L., and J. Gearhart (1981) Cell lineage analysis of cerebellar Purkinje cells in mouse chimeras. Dev. Biol. 85: 199-208.

Pilar, G., L. Landmesser, and L. Burstein (1980) Compctition for survival among developing ciliary ganglion cells. J. Neurophysiol. 43: 233-254

Sidman, R. L. (1968) Development of interneuronal connections in brains of mutant mice. In Physiological and Biochemical Aspects of Nervous Integration, S. D. Carlson, ed., pp. 163-193, Prentice-Hall, Englewood Cliffs, NJ.

Sidman, R. L. (1972) Cell interaction in the developing mammalian central nervous system. In Cell Interactions, Proc. Third Lepettit Colloq., L. G. Silvestri, ed., pp. 1-13, North-Holland, Amsterdam.

Sidman, R. L. (1983) Experimental neurogenetics. In Genetics of Neurological Disorders, R. L. Sidman and S. W. Matthysse, eds., pp. 19 46, Raven, New York.
Sidman, R. L., P. W. Lane, and M. M. Dickie (1962) Staggerer: A new mutation in the mouse affecting the cerebellum. Science 137: 610-612.

Sonmez, E., and K. Herrup (1983) Role of staggerer gene in determining cell number in cerebellar cortex. II. Granule cell death and persistence of the extcrnal granulc cell layer in young mouse chimeras. Dev. Brain Res. 12: 271-283.

Sotelo, C. (1975) Dendritic abnormalities of Purkinje cells in cerebellum of neurologic mutant mice weaver and staggerer. Adv. Neurol. 12: 335-341.

Sotelo, C., and J-P. Changeux (1974) Trans-synaptic degeneration "en cascade" in the cerebellar cortex of staggerer mutant mice. Brain Res. 67: 519-526.

Tanaka, H., and L. Landmesser (1986) Cell death of lumbosacral motoneurons in chick, quail, and chick-quail chimera embryos: A test of the quantitative matching hypothesis of neuronal cell death. J. Neurosci. 6: 2889-2899.

Tarkowski, A. K. (1961) Mouse chimeras developed from fused eggs. Nature 230: 333-334.

Wetts, R., and K. Herrup (1982a) Interaction of granule, Purkinje and olivary neurons in lurcher chimeric mice. I. Qualitative studies. J. Embryol. Exp. Morphol. 68: 87-98.

Wetts, R., and K. Herrup (1982b) Interaction of granule, Purkinje and inferior olivary neurons in lurcher chimeric mice. II. Granule cell death. Brain Res. 250: 358-362.

Wetts, R., and K. Herrup (1982c) Cerebellar Purkinje cells are descended from a small number of progenitors committed during early development: Quantitative analysis of lurcher chimeric mice. I. Neurosci. 2: 1494-1498.

Wetts, R., and K. Herrup (1983) Direct correlation between Purkinje and granule cell number in the cerebella of lurcher chimeras and wildtype mice. Dev. Brain Res. 10: 41-47.

Yoon, C. H. (1977a) Fine structure of the cerebellum of "staggererreclcr," a double mutant of mice affected by staggerer and reeler conditions. I. The premature disappearance of the external granule cell layer and ensuing cerebellar disorganization. J. Neuropathol. Exp. Neurol. 36: 413-426.

Yoon, C. H. (1977b) Fine structure of the cerebellum of "staggererreeler," a double mutant of mice affected by staggerer and reeler conditions. II. Purkinje cell anomalies. J. Neuropathol. Exp. Neurol. 36: 427-439.

Yoon, C. H. (1977c) Fine structure of the cerebellum of "staggererreeler," a double mutant of mice affected by staggerer and reeler conditions. III. Bergmann fiber anomalies. J. Neuropathol. Exp. Neurol. 36: 440-452. 